\title{
COVID-19 and neurological disorders: are neurodegenerative or neuroimmunological diseases more vulnerable?
}

\author{
Luigi Ferini-Strambi ${ }^{1,2} \cdot$ Maria Salsone $e^{2,3}$
}

Received: 6 July 2020 / Revised: 9 July 2020 / Accepted: 10 July 2020 / Published online: 21 July 2020

c) Springer-Verlag GmbH Germany, part of Springer Nature 2020

\begin{abstract}
Neurological disorders and coronavirus 2019 (COVID-19) pandemic are two conditions with a recent well-documented association. Intriguing evidences showed that COVID-19 infection can modify clinical spectrum of manifested neurological disorders but also it plays a crucial role in the development of future diseases as long-tem consequences. In this viewpoint review, we aimed to assess the vulnerability to SARS-CoV-2 infection and development of COVID-19 among neurological disorders. With this in mind, we tested the hypothesis that age rather than neuropathology itself could be decisive in neurodegenerative diseases such as Parkinson's disease, whereas neuropathology rather than age may be critical in neuroimmunological diseases such as Multiple Sclerosis. Highlighting the role of potential susceptibility or protection factors from this disastrous infection, we also stratify the risk for future neurodegeneration.
\end{abstract}

Keywords COVID-19 infection · Parkinson's disease · Alzheimer's disease $\cdot$ Multiple sclerosis

\section{Introduction}

Recent evidence links neurological disorders to coronavirus 2019 (COVID-19) pandemic, a condition which from December 2019 to today has upset our lives, becoming our world a different place. It is a novel form of human coronavirus reported for the first time in Wuhan, China which recognizes as causative agent SARS-CoV-2 (severe acute respiratory syndrome coronavirus 2) [1, 2]. SARS-CoV-2 is a nonsegmented positive-sense RNA virus belonging to the family of Coronaviridae and is the seventh coronavirus known to infect human $[3,4]$. In the context of emerging research, COVID-19 infection can exacerbate the clinical spectrum of manifested neurological diseases. On the other hand, recent findings have been thrust into the spotlight the potential role of this novel coronavirus in the future development of neurological diseases making the neurobiological

Luigi Ferini-Strambi

ferinistrambi.luigi@hsr.it

Vita-Salute San Raffaele University, Milan, Italy

2 Sleep Disorders Center, Division of Neuroscience, San Raffaele Scientific Institute, Milan, Italy

3 Institute of Molecular Bioimaging and Physiology, National Research Council, Catanzaro, Italy link between these two conditions even more interesting. This interaction, however, should not be unexpected. It is well-known that coronaviruses can be detected in the Central Nervous System (CNS) of patients with Parkinson's Disease (PD), Alzheimer Disease (AD) and multiple sclerosis (MS) [5]. Human and animal models demonstrated that also SARS-CoV-2 is able to infect the brain including the brainstem [6] entering directly through the olfactory nerves and interestingly without an initial lung involvement [7]. A possible explanation is that the infection develops as the virus glycoprotein spike binds to ACE2 (angiotensin-converting enzyme 2) receptors. These receptors are widespread in the brain, not only cardiorespiratory centers in the medulla, but also in the dopamine neurons of striatum $[8,9]$.

The existence of a close relationship between COVID19 and neurological disorders brings up some fundamental questions: first, whether the relationship is causal, specifically does one condition itself increases the incidence or morbidity/mortality of the other; second, whether and in what way COVID-19 infection modifies the clinical course of pre-existing neurological disease. Thus, it is crucial to consider not only the perspectives from which we analyze these questions but also the field of neurology in which we move, for instance neurodegenerative versus neuroimmune diseases. Considering that neurodegenerative disorders may commonly occur in elderly patients whereas that 
neuroimmune in young people, this comparison might be intriguing. From a pathogenetic perspective, it is need to investigate whether the age rather than the neuropathology itself might be a potential risk factor and vice versa. From a clinical perspective it is important to investigate whether clinical features related to the pathology, for instance rigidity respiratory system in chronic neurodegenerative diseases as Parkinson's disease (PD) may be a risk factor for the development of complications and long-term neurological sequelae. From a therapeutic perspective, it might be crucial to know whether antiviral agents such as amantadine commonly used for PD-treatment, could prevent the clinical manifestations of COVID-19 infection.

Despite significant progress made from neurologists and researchers worldwide in a very short time, several issues remain still unsolved. The main goal of this viewpoint review is to assess the vulnerability to SARS-CoV-2 infection and development of COVID-19 among neurological disorders with different pathogenesis and age-related targets such as neurodegenerative vs neuroimmunological diseases. We also highlight potential susceptibility or neuroprotective factors from this disastrous infection.

\section{COVID-19 and neurodegenerative disorders}

In this section, we will discuss the impact of SARS-CoV-2 viral infection for patients with neurodegenerative conditions with a magnifying glass on patients with movement disorders and dementias. Since SARS-CoV-2 effects on neurodegenerative, as well as neuroimmune diseases, might vary across the different pathogenesis and clinical features, we consider the evidence within three sections: (i) vulnerability to the infection; (ii) modification of the clinical course of disease, in relation to clinical neurological manifestations, disease progression and innovative strategies, to support clinicians in the management of the disease; (iii) trigger for future neurodegeneration.

\section{COVID-19 and Parkinson's disease}

There are at least two well-consolidated evidence linking COVID-19 to movement disorders, especially for PD. First, the presence of antibodies against coronavirus in the cerebrospinal fluid of patients with PD revealed more than 2 decades ago [10]. Second, the ability of coronaviruses to enter the brain through the nasal cavity causing anosmia/ hyposmia [11]. The facts that hyposmia is a common premotor feature of PD and that olfactory bulb is a selective target of the deposition of alpha-synuclein pathology [12], might to be more than just a coincidence.

\section{Vulnerability to the infection}

In a recent editorial focused on movement disorders in the world at the time of COVID-19, Jon Stoessl et al. report that to date there is no evidence that patients with movement disorders are at increased risk of coronavirus infection, compared to individuals with similar age and comorbidities [13]. To answer emerging questions on this topic Fasano et al., conducted a single-centre case-controlled survey describing clinical features/predictors of COVID-19 infection and outcome in a relatively unselected and homogeneous large cohort of PD patients from one of the largest tertiary centers of Milan, Italy [14]. They identified 105 PD patients, 32 confirmed and 73 probable cases of COVID-19. Basing on their results, COVID-19 risk, morbidity and mortality in patients with mild to moderate PD do not differ from the general population [14]. In line with these evidences, a retrospective cohort study conducted in Japan showed that, patients with Parkinsonism hospitalized for pneumonia had a lower rate of in-hospital mortality as compared to age- and sex-matched patients $[15,16]$. Contrasting results has been reported by Antonini et al., in a small simple size as PD patients of older age with longer disease duration were particularly susceptible to COVID-19 with a substantially high mortality rate [17]. Taken together, these findings suggest that although PD patients may represent a particularly vulnerable population for age-related target, respiratory muscle rigidity related to the disease, and presence of several comorbidities, PD by itself do not appears increase the risk of being infected by SARS-CoV-2 and developing COVID-19 (Fig. 1).

\section{Modification of the neurological disease and care strategies}

Eleopra et al. performed the first community-based case-control study describing the effects of symptomatic COVID-19 on motor and non-motor symptoms [17]. In their cohort consisting of 141 patients with PD resident in Lombardy, twelve COVID-19 cases had mean age and disease duration similar to the controls. Their PD patients experienced substantial worsening of both motor and non-motor symptoms (especially urinary issues and fatigue), during mild-to-moderate COVID-19 illness independently of age and disease duration. Fatigue as dominant non-motor symptom has been also reported during the SARSCoV-2 infection in PD cases described by Antonini et al., [11]. By contrast, cognitive functions were marginally involved, while none experienced autonomic failure [17]. Clinical deterioration observed in PD patients might be explained by both infection-related mechanisms and impaired pharmacokinetics of dopaminergic therapy and required therapy adjustment in one-third of cases [17].

The impact of COVID-19 on PD patients, however, cannot be restricted only to motor symptoms since indirect 


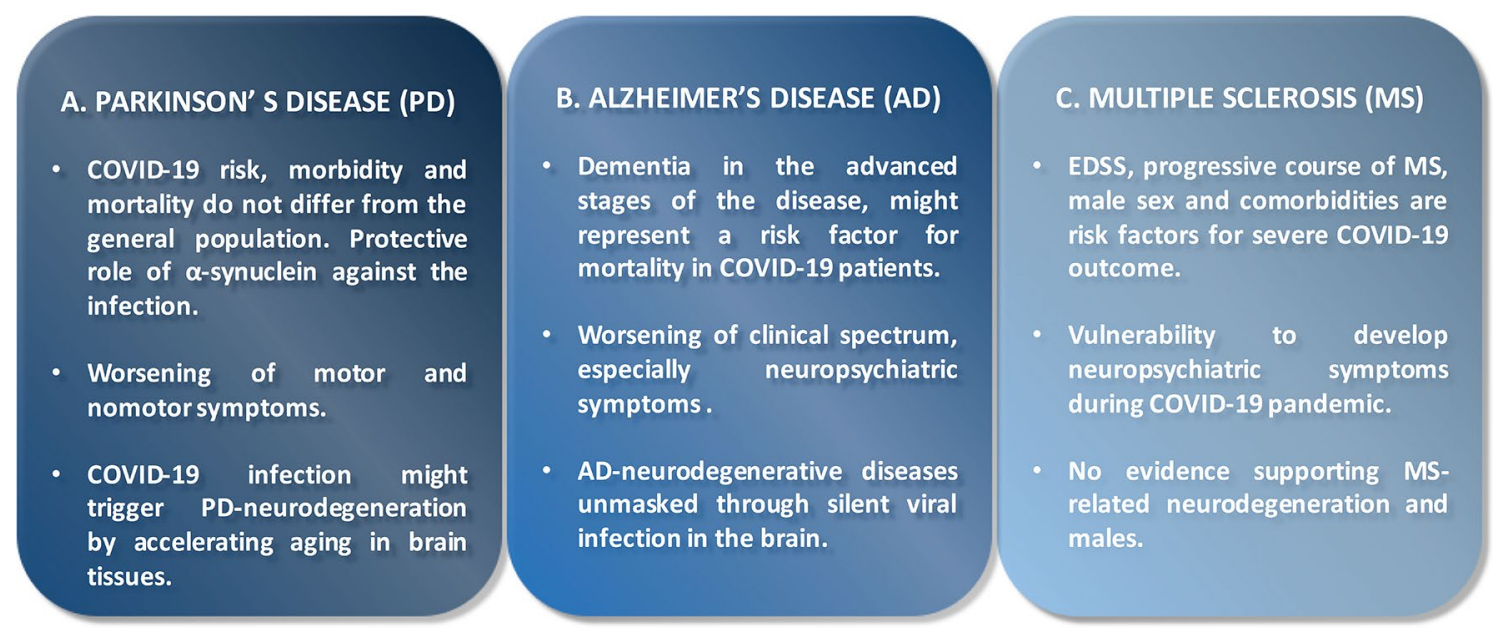

Fig. 1 Key learning points on the interaction between COVID-19 infection and Parkinson's disease (a), Alzheimer's disease (b) and multiple sclerosis (c)

effects related to the prolonged immobility under lockdown, as the impact of stress, self-isolation, social distancing and anxiety should be considered. In this context, a recent Iranian cross-sectional, case-control survey evaluated the level of anxiety among PD patients compared with caregivers and the general population [18]. Highest frequency of anxiety has been found in the PD subgroup followed by their caregivers. In addition, a strong correlation between severity of anxiety in PD patients and fear of getting COVID19 has also been reported by the authors [18]. Another consequence of COVID-19 pandemic concerns the marked reduction in physical activity [19]. This aspect should be not remiss since physical exercise may attenuate clinical symptom progression in PD patients [19-21]. In line with these evidences, Shalash et al. investigated the impact of the COVID-19 pandemic on the mental health, physical activities, and quality of life of PD patients [22]. Compared with controls, PD patients complained a negative impact on their mental health, physical activity, and health care and an interest in virtual visits [22]. In addition, Pall $\mathrm{K}$ et al., reporting the perceptions and implications of COVID-19 in 100 Indian PD patients and their caregivers, confirmed the impaired mental health, physical activity, and quality of these patients [23]. The study also highlights the importance of managing these issues and continuing care of PD patients, particularly by adopting telemedicine [23]. The fact that PD patients shows a negative impact on their mental health during lockdown period is not surprising. Sudden changes usually require a flexible adaptation to new circumstances, a condition strongly related to normal dopaminergic functioning. Indeed, PD patients may experience cognitive inflexibility, as a result of nigrostriatal dopamine depletion that forms the pathophysiological substrate of PD [24, 25].
To further complicate the precarious situation of coexistence with COVID-19 infection, there was the limited access to the routine-visits to the hospital to preserve PD patients from becoming infected. Thus, in this "new world" in which the care for our patients is significantly changed, the most important challenge for clinicians has been to reinvent their work and the greater opportunity, the telemedicine with digital-visits, e-mails or text messages, and simple telephone consultations. In this context, the Movement Disorders Society (MDS) Telemedicine Study Group has created a "step-by step" guide for assisting the movement disorders neurologists worldwide [26]. A further step forward in this direction, has been made thanks to the work of Goetz CG et al., in assessing reliability and validity of video-based MDSUnified Parkinson's Disease Rating Scale examinations compared with in-office visits [27]. Finally, digital rehabilitation (E-Rehabilitation) strategies including virtual rehabilitation platform as an alternative mode to deliver rehabilitation services at the community level [28] should be encouraged. Although the telemedicine is not superior to the quality of care with regular in-person visits, and is not yet established universally for virtual management of patients, a growing body of evidence suggests that it is associated with comparable outcomes, and offers greater efficiency and service for PD patients [29].

In summary, COVID-19 complicates the clinical course of $\mathrm{PD}$ resulting in a worsening of motor and non-motor symptoms, increased anxiety with severe complications on the quality of the life and mental health. In the COVID19 era, telemedicine has had a special role. Finally, several cross-sectional and longitudinal studies are, however, needed to better clarify the causal links between clinical and the severity of COVID-19, systemic inflammatory response with 
cytokine levels and virus detection in the cerebrospinal fluid of PD patients.

\section{Trigger for future neurodegeneration}

The fact that cortex and substantia nigra, the brain regions with higher possibilities of SARSCoV-2 penetration by ACE2 are the same associated with the most frequent neurodegenerative diseases [30] is not a simple coincidence. Lippi et al. investigated the potential role of SARS-CoV2 in the future development of neurodegenerative diseases, specifically PD [31]. A new model of neurodegeneration as SARS$\mathrm{CoV} 2$ promoting the accumulation of the alpha-synuclein (aSyn), the major protein component of Lewy bodies in the brain, has been proposed. To explain this phenomenon, the authors put the spotlight cellular pathways affected by the viral infection such as proteostasis precious in contributing to a dynamic equilibrium and activating stress response mechanisms that appear to be the same targets involved in neurodegenerative process [31]. In line with these evidences, the H1N1 infection of dopaminergic cells resulted in the formation of aSyn aggregates and not of other proteins suggesting am highly specific nature of this process [32]. In addition, in vitro models indicate that triggers alterations in proteostasis might lead to the accumulation of toxic insoluble proteins [33]. These suggestive findings indicate that SARS-CoV2 infection might trigger PD-neurodegeneration by accelerating aging in brain tissues (Fig. 2).

\section{COVID-19 and Alzheimer's disease}

In our ageing society, Dementia itself has emerged as a pandemic condition [37]. Thus, the management of a pandemic in the pandemic such as the COVID-19, brings up some concerns. First, the combination between two potential risk factors such as age and dementia for mortality in patients affected by COVID-19. Second, the complex impact of concomitant COVID-19 outbreak and dementia: the impact of confinement and social distancing on neurocognitive performance of these fragile patients, need to better investigated.

\section{Vulnerability to the infection}

It is well-documented that elderly individuals are at a higher risk for mortality after SARS-CoV-2 infection. Indeed, the estimated median age for all COVID-19 related death is 81 years, and the case fatality rate in patients aged $\geq 80$ years is $>20 \%$ [38]. Limited data are, however, available for COVID-19 in older patients, and few reports have focused on patients aged $\geq 80$ years [39-41]. All this has been reported in older patients without dementia. What happened when two potential risk factors (age and dementia) are coexisting? Covino et al. provided a risk stratification in this population [42]. Results from this single-center, retrospective, observational study, carried out in a referral center for COVID-19 in central Italy, showed that the risk of death could be not age dependent whereas severe dementia itself may be a relevant risk factor in these patients [42]. In line with these evidences, Bianchetti et al. assessed prevalence, clinical presentation and outcomes of dementia among subjects hospitalized for COVID19 infection. Data from 627 subjects admitted to Acute Hospital in Brescia province, Northern Italy were retrospectively analyzed. Compared to subjects without dementia, patients affected by dementia showed a higher mortality about $40 \%$ [43]. Taken together, these finding suggest dementia, especially in the advanced stages of the disease, might represent an important risk factor for mortality in COVID-19 patients (Fig. 1).

\section{Modification of the neurological disease and care strategies}

Patients with AD patients are vulnerable to disasters and crisis, because of their neurocognitive impairments and rich neuropsychiatric symptomatology. This is especially true during a humanitarian crisis such as COVID-19 pandemic. About the $80 \%$ of AD patients may exhibit at least one neuropsychiatric symptom over the course of their disease [44].
Fig. 2 Title: COVID-19 infection and Neurodegeneration. Legend: schematic representation of main targets and pathogenetic mechanisms linking COVID-19 infection to Parkinson's disease and Alzheimer's disease neurodegeneration

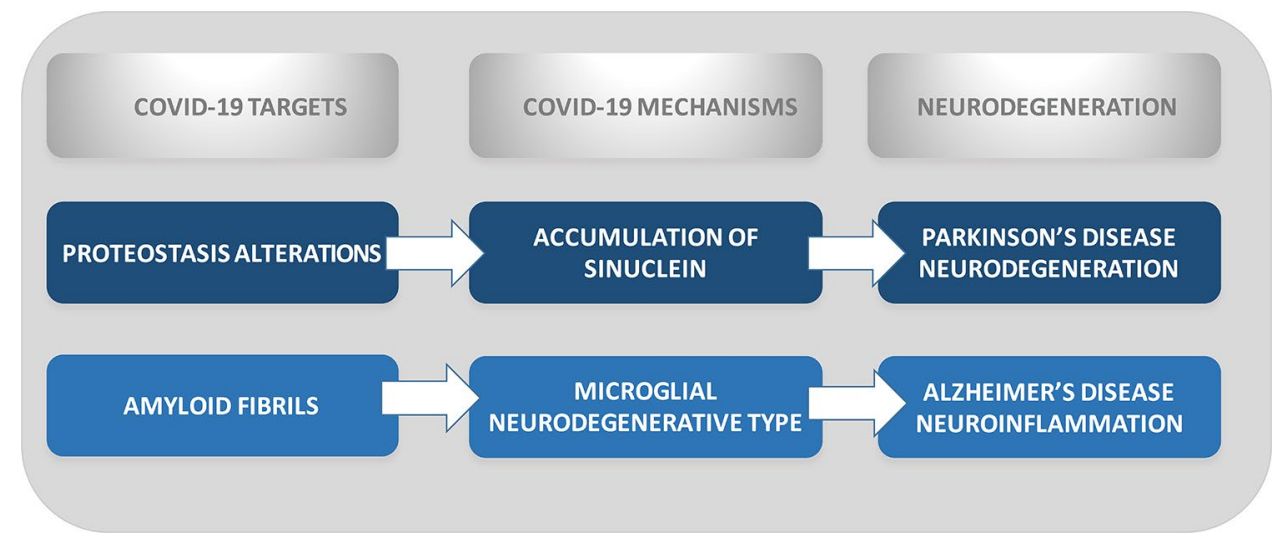


These features are typically fluctuating, emerging in more advanced $\mathrm{AD}$, even if can also manifest in early the prodromal stages [45]. Indeed, among the AD clinical spectrum, neuropsychiatric symptoms including depression, anxiety, apathy, agitation, and hallucinations appear to be subjected to a sudden deterioration. Moreover, it should also be considered the consequences of these features: (i) increased rate of disease progression and institutionalization; (ii) alteration of the treatment responses and prognosis [44]; (iii) decrease of the patients' quality of life [46]. To investigate the occurrence and severity of neuropsychiatric symptoms during the COVID-19 confinement is need. In line with this, Boutoleau-Bretonniere et al. offered the first investigation of the effects of confinement during the COVID-19 crisis on neuropsychiatric symptoms in AD [45]. Their results showed that only about $30 \%$ of AD patients demonstrated neuropsychiatric changes during the confinement. The duration of confinement significantly correlated with the severity of symptoms, as well as with their caregivers' distress. Interestingly, the confinement exacerbated neuropsychiatric symptoms in patients with low cognitive function in $\mathrm{AD}$, whereas no such symptoms were induced in patients with more preserved cognition [45]. In line with these evidences, other authors demonstrated the worsening of neuropsychiatric symptoms, with agitation, apathy and aberrant motor activity being the most affected symptoms, in $\mathrm{AD}$ and MCI during 5 weeks of lockdown in Spain [47]. Concerning the clinical presentation of COVID-19 in subjects with dementia, there is concordance that it may be atypical, especially in most advanced and severe diseases thus reducing early recognition of symptoms and hospitalization. Indeed, in patients with AD the classic symptoms of COVID-19 infection such as fever, dyspnea and cough were less frequent, while they mainly experienced diarrhea or drowsiness [42, 48]. Finally, delirium caused by hypoxia, a prominent clinical feature of COVID-19, could complicate the presentation of dementia thus needing for dementia care and support [49, 50]. Despite the preliminary nature, these findings confirmed the expected worsening of clinical spectrum, especially neuropsychiatric symptoms during COVID-19 pandemic in AD patients.

Considering the complex nature of the interaction between COVID-19 and dementia, the international recommendations suggest to provide worldwide support for patients with dementia. Multidisciplinary teams, as well as a digital revolution are urgent need. Responding to this need, the American Academy of Neurology has also developed a guidance for clinicians and practices to implement telemedicine services amid the COVID-19 crisis [51, 52]. Cuffaro et al. [52] recently suggest that telemedicine and digital technology devices, including smartphones can be really helpful in remote monitoring and care of people with Dementia. Moreover, technological devices as videoconference or smartphone apps might be used for follow-up visits and support to patients and to acquire digital markers of clinical progression of the disease [52]. Whether tele-rehabilitation platforms for neurorehabilitation care including physical, language and cognitive rehabilitation, exergaming, with remote supervision will be offered, this emergency will produce long-term healthcare positive [53].

In summary, despite the preliminary nature these findings confirmed the expected worsening of clinical spectrum, especially neuropsychiatric symptoms during COVID-19 pandemic in AD patients. A digital revolution to support the clinicians in the management of these fragile patients is need.

\section{Trigger for future neurodegeneration}

Although the long-term implications of SARS-CoV-2 and its effects on the brain are not well known, its potential role in the future neurodegeneration may be of importance to the field of AD research (Fig. 2). Severe outcomes after SARSCoV-2 infection are often associated with a "cytokine storm" of pronounced inflammation causing an increase of proinflammatory cytokines such interleukin-1 (IL-1), and IL-6 [54]. In $\mathrm{AD}$ patients, this may synergize with amyloid-stimulated Type I interferon (IFN) response thus creating the "perfect storm" [54]. This might explain why pre-symptomatic people with undiagnosed AD may see an acceleration of symptoms due to a bout of systemic inflammation resulting from SARS-CoV-2 infection [54]. In addition, other authors hypothesized that affected patients may be at higher risk of developing cognitive decline after overcoming the primary COVID-19 infection [55]. Pathogenetically, this may result from direct negative effects of the immune reaction, acceleration or aggravation of pre-existing cognitive deficits, or de novo induction of a neurodegenerative disease. On the basis of these findings, it possible to speculate that there may be a population at risk to develop neurodegenerative diseases unmasked through silent viral infection in the brain.

\section{COVID-19 and neuroimmunological disorders}

In the context of emerging COVID-19 pandemic, an urgent attention should be focused on a population of particular interest such as that neuroimmunological disorders. In this section, we will discuss the impact of the SARS-CoV-2 viral infection for patients with these conditions with a magnifying glass on patients with Multiple Sclerosis (MS). No consistent data are to date available for other neuroimmunological disorders such as neuromyelitis optica spectrum disorders, Guillain-Barré syndrome or chronic dysimmune neuropathies. 


\section{COVID-19 and multiple sclerosis}

It is not surprising that MS may represent a population of particular interest: first, for the immunological nature itself of the disease; second, for the clinical management regarding the disease-modifying therapies (DMTs) able to alter the immune functions and thus increase the susceptibility COVID-19. The early identification of potential risk factors becomes crucial to identify an individual strategy concerning the clinical management of these critical patients during the COVID-19 pandemic.

\section{Vulnerability to the infection}

The relationship between COVID-19 and MS is complex. The coronavirus family has been previously investigated for a potential association with MS, and more recently has been utilised to make a mouse model of the disease [58]. It has further been complex the challenge for neurologists worldwide to stratify the risk of the viral infection in MS patients, especially those receiving immunosuppressant or immunomodulatory therapy. Although it has been documented that MS patients may theoretically have an increased risk of the infections compared with the general population, it remains until debated whether MS patients are or not at high-risk to be infected by SARS-CoV-2 and to develop COVID-19. In a recent month's journal club, Willis MD et al. considered three papers relevant to answer these questions [58]. The first study aimed to characterize the infection risk of patients with MS compared with a cohort of patients without MS, using two large databases with a long followup time $[58,59]$. According with the results, MS patients were demonstrated to be at an increased risk generally of infections, and of infections requiring hospitalization. The second study aimed to examine the risk of serious infections associated with routinely used MS DMTs as well as rituximab, which is commonly used in this population [58, 60]. MS patients treated with DMTs are at a generally increased risk of infections, with rituximab associated with the highest rate of serious infections [58]. In addition, a populationbased retrospective cohort study investigated the association between MS DMTs and risk of infections [61]. Their main results demonstrated that the exposure to a second generation DMT was associated with an increase in the risk while of first generation were not associated. Of note, with IFN- $\beta$ was associated with a lower risk of pneumonia. In line with this preliminary evidence, Fan $\mathrm{M}$ et al. reported no increased risk of COVID-19 infection was MS or NMOSD, irrespective of whether these patients received DMDs. A possible explanation for this phenomenon is that stringent preventive measures adopted by neurologists to reduce COVID-19 infection in these patients may have contributed to low risk of COVID-19 infection [62]. Finally, data from the World
Health Organization, has not yet been translated in relation to this cohort of COVID-19 patients who are on DMT [63]. Louapre et al. very recently also reported that age, EDSS, progressive course of MS, male sex and comorbidities including cardiovascular and pulmonary diseases, diabetes, and obesity, were identified as risk factors [64]. The presence of DMT, however, was not associated with severity of COVID-19 infection having a lower risk of hospitalization in their univariate analysis [64]. Taken together, these findings suggest that, although it has initially been reported an increased risk to COVID-19 infection in MS patients, to date the risk estimate for the MS patients remains until debated.

\section{Modification of the neurological disease and care strategies}

Results from a pilot phase of an investigation of COVID-19 among people with MS, based on a core set of data collected on 232 patients from 38 centers, indicated that the severity of COVID-19 infection classified as mild (no pneumonia or mild pneumonia) was present in about $96 \%$ of the MS patients [65]. Louapre et al. also described the clinical profile and outcomes in patients with MS and COVID-19. These authors performed a multicenter, retrospective observational cohort study in $347 \mathrm{MS}$ patients presenting with a confirmed or highly suspected diagnosis of COVID-19 between March 1, 2020, and May 21, 2020 [64]. Their main outcome was COVID-19 severity assessed on a 7-point ordinal scale [ranging from 1 (not hospitalized with no limitations on activities) to 7 (death)] with a cut off at 3 (hospitalized and not requiring supplemental oxygen). Seventy-three patients had a COVID-19 severity score of 3 or more. As occurring in general population, fever and dyspnea were more common in MS patients hospitalized for COVID-19 while anosmia/ageusia and headache were more common in patients who were not hospitalized [64]. Considering that cognitive dysfunction usually affects up to $70 \%$ of MS patients, it is not surprising that these patients are vulnerable to develop neuropsychiatric symptoms during COVID-19 pandemic [66]. Thus, MS specialists should adapt a strategy to monitor MS patients for neuropsychiatric complications and also opt for applicable treatment options [66]. Acute and long term effects of COVID-19 on disease course of MS population should a subject for future research. Now, it is also the time of resilience: this disastrous pandemic may be a great opportunity for databases promoting MS research and collaboration [67].

Considering the clinical relevance of this issue and that, Italy was the first European country involved in a COVID-19 pandemic, the Italian Multiple Sclerosis Society (AISM), the Italian Multiple Sclerosis Foundation (FISM), and the Multiple Sclerosis Study Group of the Italian Neurological Society have set up a program to help with these crucial elements in the response to COVID-19 in patients MS 
[65]. Regarding the clinical management of these critical patients, the risks and benefits of immunoactive treatments and adjustments to these treatments must be assessed [68]. In this suggestive picture the protective role of the first-generation DMTs such as interferon-beta (IFN- $\beta$ ) is emerging [69]. Future studies, however, to examine the efficacy of interferon IFN- $\beta$ alone or in combination with other drugs to treat severe or critically ill patients with confirmed COVID19 compared with placebo are warranted [69]. An interesting window in COVID-19 era is opened on the teriflunomide in MS. Recent observations suggest that teriflunomide may not need to be discontinued in MS patients who develop an active COVID-19 infection [70]. Finally, the special role of telemedicine in COVID-19 pandemic emerged especially in the management of MS patients. Bonavita $\mathrm{S}$ et al. proposed a possible approach for the remote monitoring of infection risk in people with MS, especially those on immunosuppressant drugs, during the pandemic. This tool will also limit unnecessary accesses to the MS centers reducing the risk of spreading the infection [71].

\section{Trigger for future neurodegeneration}

Despite the existence of a close relation between neuroinflammation and neurodegeneration in MS brains [72], to date no enough evidence is available regarding the long-term implications of SARS-CoV-2 as potential role in the future neurodegeneration in these patients.

\section{Discussion}

The COVID-19 pandemic, a novel condition that in this few months has been completely storming the planet, brings with it an intense trail of mysteries. First of all, the reason why some subjects are completely asymptomatic while others develop deadly consequences. Second, the reason why some neurological diseases are more vulnerable than others to contracting the virus and developing nefarious complications. There necessarily is something that protects and similarly something that damages. With this in mind, we identified among neurological conditions with different pathogenesis and age-related targets, the protection and susceptibility risk factors against this global pandemic.

It is singular that COVID-19 risk, morbidity and mortality in $\mathrm{PD}$, a population with an intrinsically increased vulnerability for many risk factors, such as age, respiratory muscle rigidity and comorbidities, do not differ from the general population. Whether this is true, we can speculate that PD neuropathology itself might exercise a neuroprotective effect against COVID-19 infection for some reasons. Firstly, the modality of brain penetration of SARS-CoV-2 could be considered. It is well-known that the infection develops as the virus spike binds to ACE2 receptors, highly expressed in the dopamine neurons of striatum $[8,9,30]$. It is equally well-documented that PD patients show reduced nigrostriatal dopamine neurons of substantia nigra as result of the Lewy-body neurodegeneration. It is likely that the SARS$\mathrm{CoV} 2$ neuroinvasion may be reduced in these patients. Secondly, the protective role of $\alpha$-synuclein against the SARSCoV-2 infections can be considered [34]. It has been recently reported that $\alpha$-synuclein plays a crucial role in the COVID19 infection: by exercising inhibiting the viral neuroinvasion, by offering protection against proinflammatory responses, by facilitating immune reactions against infections being implicated in microglia process $[3,34]$. Thirdly, the neurobiology of SARS-CoV-2 infection in PD can be considered. GomezPinedo et al. reviewed some pathological cases COVID-19 reporting no gliosis, microgliosis, and markers of inflammatory signs in brain tissue while the virus was observed inside vacuoles and/or inclusions [30]. Vacuoles containing virus have been reported in MERS-CoV and SARS-CoV infection [35]. Finally, PD patients may potentially protected by the specific treatment such as amantadine, a drug for years used for the treatment of influenza. It able to inhibit the viral replication by blockade of the influenza M2 ion channel required to deliver viral ribonucleoprotein into the host cytoplasm [13]. Although the therapeutic implications of amantadine are unknown, it also appears block a pore in the envelope protein of SARS-CoV [36].

Differently from PD patients, that with AD show a higher mortality compared to subjects without dementia. This phenomenon, however, could be not age dependent and identifies other causes: first, the AD neuropathology itself. Amyloid fibrils may entrap viral particles, pathogens and subsequently leading to further activation of the "microglial neurodegenerative phenotype" [54]. This subset of microglia causes an increased expression of IFN pathway, a crucial component both in AD and COVID-19 infection. Indeed, IFN was found to stimulate complement cascade activation and promote synapse elimination with an enhancement of the immune response [54]. In this viewpoint, the suppression of IFN response in both AD and COVID-19 (or comorbidity of the two) might a potential strategy for controlling the excessive immune response [54]. Second possible cause is the presence of comorbidities. Individuals with dementia are more likely to have cardiovascular disease, diabetes, and pneumonia compared to individuals of the same age without dementia [55]. In particular, the co-occurrence of obesity and type 2 diabetes could place these populations at an increased risk for severe COVID-19 pathology and mortality [56]. To corroborate this hypothesis, a consumption of a diet rich in fat and refined carbohydrates and sugars, and low levels of fibers activate the innate immune system and impairs adaptive immunity, leading to chronic inflammation and impaired host defense against viruses [56]. Third possible 
cause is the social behavior of AD patients. Indeed, individuals with dementia are unable to follow the recommendations from public health authorities to reduce the transmission of COVID-19: hand hygiene, wearing masks, covering one's mouth and nose when coughing, maintaining physical distance from others, ignoring the warnings and lacking sufficient, could expose them to higher chance of infection [50, 57]. Thus, it is early to know whether dementia itself may be considered a potential risk factor for COVID-19 infection because the presence of confounding factors linked to the inconsistent social behaviors and comorbidities.

From a theoretical perspective, it is believable that COVID 19 pandemic virus may exacerbate MS disease. With the aim to discuss recent data indicating a strict correlation especially between EDSS and age and COVID-19 severity and no association with DMTs exposure, some reflections are due. First, the immunological nature of MS disease and COVID-19 infection. In the context of emerging literature, EDSS has been reported associated with the highest variability of COVID-19 severe outcome [64]. A possible explanation is that morbidity and mortality in COVID-19 can result from an overlapping between immune response caused by the virus and the immunological state of the subjects. To block the viral infection, both innate and adaptive immune responses are really crucial. Viral infection is prevented through innate immunity with type I interferons inhibit and natural killer (NK) cells while is combatting through the adaptive immune response with immunity generated by antibodies and cytotoxic T lymphocytes (CTL), especially $\mathrm{CD} 8^{+} \mathrm{T}$ [73]. Thus, it is possible to speculate that in patients with pre-existing impaired regulation of immune responses such as MS, COVID-19 infection may trigger a further amplification of immune pathways. Second, age and COVID-19 infection. Age has been reported to be associate with the highest variability of COVID-19 severe outcome [64]. Age, however, was identified as a major risk factor for the severity of COVID-19 infection. Thus it is not surprising that also in MS population consisting of a substantial number of individuals with MS are older than 60 years, it is independent risk factors for COVID-19 severity [64]. Third, DMTs and COVID-19 infection. Do these drugs have a harmful or protective effect? DMTs limit the immune response, in theory, thus allow for greater viral replication and potentially worse infection. On the other hand, limiting cytokine storm and the exaggerated immune response induced by SARS-CoV-2 infection, these drugs may have some protective and beneficial effects against this novel virus. In addition, most DMTs do not particularly target the innate immune system and few have any major long-term impact on $\mathrm{CD}^{+} \mathrm{T}$ cells to limit protection against COVID19 [74]. There are not, however, enough data on DMT to indicate about susceptibility or protection from CIVODinfection of these drugs in MS. Other important point to consider in future, is whether/how DMT affect responses to vaccination, as the ultimate measure to contain the pandemic. Overall, we can summarize that despite the risk estimate of COVID-19 complications on young people with MS patients remains until debated, attention should be placed in older MS patients with a progressive course of the disease.

The second outcome of our investigation has been to stratify the risk for future neurodegeneration as long-term sequelae of COVID-19 infection. PD and AD patients showed salient differences concerning the brain neuroinvasion (reduced ACE receptors in PD) and neurobiology of the virus (amyloid fibrils able to capture viral particles), but also on the pathogenic mechanisms leading to future neurodegeneration. While SARS-CoV2 infection triggers PDneurodegeneration by accelerating aging and accumulation of abnormal proteins in brain tissues, the amyloid-stimulated IFN response resulting in neuroinflammation would be the basis of AD-neurodegeneration. This is not surprising since the association between pathological levels of neuroinflammation and $\mathrm{AD}$ and other forms of dementia is well-known. These findings represent an important advantage in the future, opening a novel therapeutic window: $\mathrm{AD}$ treatments increasing acetylcholine levels has been proposed in controlling inflammation and preventing a "cytokine storm" after SARS-CoV-2 infection [54], thus reducing the risk of development AD-neuropathology over the time. Finally, no enough evidence is available regarding the COVID-19 implications in MS related-neurodegeneration.

In this review, we asked whether age rather than neuropathology itself might make more vulnerable a specific neurological disease to COVID-19 infection, especially neurodegenerative versus neuroimmunological disorders. Thus, we entered in an intriguing but select research window and this might represent a limitation of the study. However, we are confident that most of the existing literature concerning the COVID-19 impact on neurological disorders has been examined.

\section{Concluding remarks}

The most important clinical lesson we have learned from COVID-19 in neurological disorders is that COVID-19 is really a completely unpredictable condition. We found that PD neuropathology itself may be a protective factor against the SARS-CoV-2 infection, whereas age and progressive course of the disease are potential risk factors in MS disease. Thus the final answer to our initial question is that MS population might more vulnerable to COVID-19 severe outcome rather that neurodegenerative. Conversely, future neurodegeneration as long-term complications of COVID-19 infections may like occur in neurodegenerative population. A large cohort of neurological patients experiencing a severe 
acute respiratory syndrome is need to confirm our suggestive conclusions.

Acknowledgement No funding was received for this study.

Author contributions All authors contributed to the study concept and design, to data acquisition and analysis, and to drafting manuscript and figures.

\section{Compliance with ethical standards}

Conflicts of interest The authors declare that they have no competing interests.

\section{References}

1. Wu F, Zhao S, Yu B et al (2020) A new coronavirus associated with human respiratory disease in China. Nature 579:265-269

2. Chen X, Yu B (2020) First two months of the 2019 coronavirus disease (COVID-19) epidemic in China: real-time surveillance and evaluation with a second derivative model. Glob Health Res Policy 5:7

3. Lippi A, Domingues R, Setz C, Outeiro TF, Krisko A (2020) SARS-CoV-2: at the crossroad between aging and neurodegeneration. Mov Dis. https://doi.org/10.1002/mds.28084

4. Corman VM, Muth D, Niemeyer D, Drosten C (2018) Hosts and sources of endemic human coronaviruses. Adv Virus Res 100:163-188

5. Matías-Guiu J, Gomez-Pinedo U, Montero-Escribano P, GomezIglesias P, Porta-Etessam J, Matias-Guiu JA (2020) Should we expect neurological symptoms in the SARS-CoV-2 Epidemic? Neurologia 35:170-175. https://doi.org/10.1016/j.nrl.2020.03.001

6. Li YC, Bai WZ, Hashikawa T (2020) The neuroinvasive potential of SARS-CoV2 may play a role in the respiratory failure of COVID-19 patients. J Med Virol. https://doi.org/10.1002/ jmv. 25728[Epub ahead of print].

7. Li K, Wohlford-Lenane C, Perlman S et al (2016) Middle East respiratory syndrome coronavirus causes multiple organ damage and lethal disease in mice transgenic for human dipeptidyl peptidase 4. J Infect Dis 213:712-722

8. Doobay MF, Talman LS, Obr TD, Tian X, Davisson RL, Lazartigues E (2007) Differential expression of neuronal ACE2 in transgenic mice with overexpression of the brain renin-angiotensin system. Am J Physiol Regul Integr Comp Physiol 292:R373-R381

9. Rodriguez-Perez AI, Garrido-Gil P, Pedrosa MA et al (2019) Angiotensin type 2 receptors: role in aging and neuroinflammation in the substantia nigra. Brain Behav Iimmun S0889-1591(19):31128-31136

10. Fazzini E, Fleming J, Fahn S (1992) Cerebrospinal fluid antibodies to coronavirus in patients with Parkinson's disease. Mov Disord 7(2):153-158

11. Antonini A, Leta V, Teo J, Chaudhuri KR (2020) Outcome of Parkinson's disease patients affected by COVID-19. Mov Disord 35(6):905-908

12. Braak H, Del Tredici K, Rub U et al (2003) Staging of brain pathology related to sporadic Parkinson's disease. Neurobiol Aging 24:197-211

13. Stoessl J, Bhatia KP, Merello M (2020) Movement disorders in the world of COVID-19. Mov Disord Clin Pract 7(4):355-356

14. Fasano A, Antonini A, Katzenschlager R et al (2020) Management of advanced therapies in Parkinson's disease patients in times of humanitarian crisis: the COVID-19 experience. Mov Disord Clini Pract 7(4):361-372

15. Papa SM, Brundin P, Fung VSC et al (2020) Impact of the COVID-19 pandemic on Parkinson's disease and movement disorders. Mov Disord Clin Pract 7(4):357-360

16. Jo T, Yasunaga H, Michihata N et al (2018) Influence of Parkinsonism on outcomes of elderly pneumonia patients. Parkinsonism Relat Disord 54:25-29

17. Cilia R, Bonvegna S, Straccia G, Andreasi NG, Elia AE, Romito LM et al (2020) Effects of COVID-19 on Parkinson's disease clinical features: a community-based case-control study. Mov Disord. https://doi.org/10.1002/mds.28170Online ahead of print

18. Salari M, Zali A, Ashrafi F, Etemadifar M, Sharma S, Hajizadeh $\mathrm{N}$, Ashourizadeh $\mathrm{H}$ et al (2020) Incidence of anxiety in parkinson's disease during the coronavirus disease (COVID-19) pandemic. Mov Disord. https://doi.org/10.1002/mds.28116Online ahead of print

19. Helmich RC, Bloem BR (2020) The impact of the COVID-19 pandemic on Parkinson's disease: hidden sorrows and emerging opportunities. J Parkinsons Dis 10(2):351-354

20. Schenkman M, Moore CG, Kohrt WM, Hall DA, Delitto A, Comella CL et al (2018) Effect of high-intensity treadmill exercise on motor symptoms in patients with de novo Parkinson disease. JAMA Neurol 75:218-219

21. van der Kolk NM, de Vries NM, Kessels RPC, Joosten H, Zwinderman AH, Post B et al (2019) Effectiveness of home-based and remotely supervised aerobic exercise in Parkinson's disease: adouble-blind, randomised controlled trial. Lancet Neurol 18:998-1008

22. Shalash A, Roushdy T, Essam M, Fathy M, Dawood NL, Abushady EM et al (2020) Mental health, physical activity, and quality of life in Parkinson's disease during COVID-19 pandemic. Mov Disord. https://doi.org/10.1002/mds.28134

23. Prasad S, Holla VV, Neeraja K et al (2020) Parkinson's disease and COVID-19: perceptions and implications in patients and caregivers. Mov Disord. https://doi.org/10.1002/mds.28088

24. Helmich RC, Aarts E, De Lange FP, Bloem BR, Toni I (2009) Increased dependence of action selection on recent motor history in Parkinson's disease. J Neurosci 29:6105-6113

25. Robbins TW, Cools R (2014) Cognitive deficits in Parkinson's disease: a cognitive neuroscience perspective. Mov Disord 29:597-607

26. https://www.movementdisorders.org/MDS/About/Commit eesOtherGroups/Telemedicine-in-Your-Movement-Disorders-Practice-A-Step-by-Step-Guide, Accessed April 179 26, 2020.

27. Goetz CG, Stebbins GT, Luo S (2020) Movement disorder societyunified Parkinson's disease rating scale use in the covid-19 Era. Mov Disord 35(6):911. https://doi.org/10.1002/mds.28094

28. Srivastav AK, Samuel AJ (2020) E-Rehabilitation: One Solution for Patients With Parkinson's Disease in COVID-19 Era. Parkinsonism Relat Disord. https://doi.org/10.1016/j.parkreldis 2020.05.021 Online ahead of print

29. Cubo E, Hassan A, Bloem BR, Mari Z, MDS-Telemedicine Study Group (2020) Implementation of telemedicine for urgent and ongoing healthcare for patients With Parkinson's disease during the COVID-19 pandemic: new expectations for the future. J Parkinsons Dis. https://doi.org/10.3233/JPD-202108

30. Gomez-Pinedo U, Matias-Guiu J, Sanclemente Alaman I, MorenoJimenez L, Montero-Escribano P, Matias-Guiu JA (2020) Is the brain a reservoir organ for SARS2-CoV2? J Med Virol. https:// doi.org/10.1002/Jmv.26046

31. Marreiros R, Müller-Schiffmann A, Trossbach SV et al (2020) Disruption of cellular proteostasis by H1N1 influenza A virus causes $\alpha$-synuclein aggregation. Proc Natl Acad Sci USA 117:6741-6751 
32. Liu XD, Ko S, Xu Y et al (2012) Transient aggregation of ubiquitinated proteins is a cytosolic unfolded protein response to inflammation and endoplasmic reticulum stress. J Biol Chem 287:19687-19698

33. Wahmane SA, Achbani A, Ouhaz Z, Elatiqi M, Belmouden A, Nejmeddine $M(2020)$ The possible protective role of $\alpha$-synuclein against the SARS-CoV-2 infections in patients with Parkinson's disease. v Disord. https://doi.org/10.1002/mds.28185.

34. Austin SA, Rojanathammanee L, Golovko MY, Murphy EJ, Combs CK (2011) Lack of alpha-synuclein modulates microglial phenotype in vitro. Neurochem Res 36:994-1004

35. Ulasli M, Verheije MH, de Haan CAM, Reggiori F (2010) Qualitative and quantitative ultrastructural analysis of the membrane rearrangements induced by coronavirus. Cell Microbiol 12:844-861. https://doi.org/10.1111/j.1462-5822.2010.01437.x

36. Torres J, Maheswari U, Parthasarathy K, Ng L, Liu DX, Gong X (2007) Conductance and amantadine binding of a pore formed by a lysineflanked transmembrane domain of SARS coronavirus envelope protein. Protein Sci 16:2065-2071

37. Fox NC, Petersen RC (2013) The G8 dementia research summita starter for eight? Lancet 382:1968-69 5 US Centers for Disease Control

38. Onder G, Rezza G, Brusaferro S (2020) Case-fatality rate and characteristics of patients dying in relation to COVID-19 in Italy. JAMA 323(18):1775-1776

39. Liu K, Chen Y, Lin R, Han K (2020) Clinical features of COVID19 in elderly patients: a comparison with young and middle-aged patients. J Infect 80(6):e14-e18

40. Leung C (2020) Risk factors for predicting mortality in elderly patients with COVID-19: a review of clinical data in China. Mech Ageing Dev 118:111255

41. Wang L, He W, Yu X et al (2020) Coronavirus disease 2019 in elderly patients: characteristics and prognostic factors based on 4-week follow-up. J Infect 6:639-645

42. Covino M, De Matteis G, Santoro M, Sabia L, Simeoni B, Candelli $\mathrm{M}$ et al (2020) Clinical characteristics and prognostic factors in COVID-19 patients aged $\geq 80$ years. Geriatr Gerontol Int. https ://doi.org/10.1111/ggi.13960

43. Bianchetti A, Rozzini R, Guerini F, Boffelli S, Ranieri P, Minelli $G$ et al (2020) Clinical presentation of COVID19 in dementia patients. J Nutr Health Aging 24(6):560-562

44. Lyketsos CG, Sheppard J-ME, Steinberg M, Tschanz JAT, Norton MC, Steffens DC et al (2001) Neuropsychiatric disturbance in Alzheimer's disease clusters into three groups: the cache county study. Int J Geriatr Psychiatry 16:1043-1053

45. Boutoleau-Bretonnière $\mathrm{C}$, Pouclet-Courtemanche $\mathrm{H}$, Gillet $\mathrm{A}$, Bernard A, Deruet AL, Gouraud I et al (2020) The effects of confinement on neuropsychiatric symptoms in Alzheimer's disease during the COVID-19 crisis. J Alzheimers Dis. https://doi. org/10.3233/JAD-200604

46. Lanct ${ }^{\wedge}$ ot KL, Amatniek J, Ancoli-Israel S, Arnold SE, Ballard C, Cohen-Mansfield J et al (2017) Neuropsychiatric signs and symptoms of Alzheimer's disease: new treatment paradigms. Alzheimers Dement 3:440-449

47. Lara B, Carnes A, Dakterzada F, Benitez I, Piñol-Ripoll G (2020) Neuropsychiatric symptoms and quality of life in spanish patients with Alzheimer's disease during the COVID-19 lockdown. Eur J Neurol. https://doi.org/10.1111/ene.14339

48. Isaia G, Marinello R, Tibaldi V, Tamone C, Bo M (2020) Atypical presentation of covid-19 in an older adult with severe Alzheimer disease. Am J Giatr Psychiatry 28(7):790-791. https://doi. org/10.1016/j.jagp.2020.04.018

49. Marcantonio ER (2017) Delirium in hospitalized older adults. N Engl J Med 377:1456-1466

50. Wang H, Li T, Barbarino P, Gauthier S, Brodaty H, Molinuevo JL, Xie $\mathrm{H}$ et al (2020) Dementia care during COVID-19.
Lancet 395(10231):1190-1191. https://doi.org/10.1016/S0140 -6736(20)30755-8Epub 2020 Mar 30

51. Hatcher-Martin JM, Adams JL, Anderson ER, Bove R, Burrus TM, Chehrenama M et al (2020) Telemedicine in neurology: telemedicine work Group of the American Academy of neurology update. Neurology 94:30-38

52. Cuffaro L, Di Lorenzo F, Bonavita S, Tedeschi G, Leocani L, Lavorgna L (2020) Dementia care and COVID-19 pandemic: a necessary digital revolution. Neurol Sci 17:1-3

53. Leocani L, Diserens K, Moccia M, Caltagirone C (2020) Disability through COVID-19 pandemic: neurorehabilitation cannot wait. Eur J Neurol. https://doi.org/10.1111/ene.14320Online ahead of print

54. Naughton SX, Raval U, Pasinetti GM et al (2020) Potential novel role of COVID-19 in Alzheimer's disease and preventative mitigation strategies. J Alzheimers Dis. https://doi.org/10.3233/JAD200537Online ahead of print

55. Bauer K, Schwarzkopf L, Graessel E et al (2014) A claims databased comparison of comorbidity in individuals with and without dementia. BMC Geriatr 14:1-13

56. Butler MJ (2020) Barrientos RMThe impact of nutrition on COVID-19 susceptibility and long-term consequences. Review Brain Behav Immun 87:53-54. https://doi.org/10.1016/j. bbi.2020.04.040Epub 2020 Apr 18

57. Brown E, Kumar S, Rajji TK, Pollock BP, Mulsant BH (2020) Anticipating and mitigating the impact of the COVID-19 pandemic on alzheimer's disease and related dementias. Am J Geriatr Psychiatry. https://doi.org/10.1016/j.jagp.2020.04.010Online ahead of print

58. Willis MD, Robertson NP (2020) Multiple sclerosis and the risk of infection: considerations in the threat of the novel coronavirus, COVID-19/SARS-CoV-2. J Neurol 267:1567-1569

59. Persson R, Lee S, Ulcickas Yood M, Wagner CAPTM, Minton $N$, Niemcryk $S$ et al (2020) Infections in patients diagnosed with multiple sclerosis: A multi-database study. Mult Scler Relat Disord 41:101-982

60. Luna G, Alping P, Burman J, Fink K, Fogdell-Hahn A, Gunnarsson $\mathrm{M}$ et al (2019) Infection risks among patients with multiple sclerosis treated with fingolimod, natalizumab, rituximab, and injectable therapies. JAMA Neurol. https://doi.org/10.1001/jaman eurol.2019.3365[Epub ahead of print]

61. Wijnands JMA, Zhu F, Kingwell E, Fisk JD, Evans E, Marrie RA et al (2018) Disease-modifying drugs for multiple sclerosis and infection risk: a cohort study. J Neurol Neurosurg Psychiatry 89:1050-1056

62. Fan M, Qiu W, Bu B, Xu Y, Yang H, Huang D et al (2020) Risk of COVID-19 infection in MS and neuromyelitis optica spectrum disorders. Neurol Neuroimmunol Neuroinflamm 7:e787. https:// doi.org/10.1212/NXI.0000000000000787

63. Mansoor S, Kelly S, Murphy K, Waters A, Siddiqui NS (2020) COVID-19 pandemic and the risk of infection in multiple sclerosis patients on disease modifying therapies: "what the bleep do we know?". Egypt J Neurol Psychiatr Neurosurg. 56(1):44

64. Louapre C, Collongues N, Stankoff B, Giannesini C, Papeix C, Bensa C (2020) Clinical characteristics and outcomes in patients with coronavirus disease 2019 and multiple sclerosis. JAMA Neurology. https://doi.org/10.1001/jamaneurol.2020.2581Online ahead of print

65. Sormani MP (2020) Italian study group on COVID-19 infection in multiple sclerosis: an Italian programme for COVID-19 infection in multiple sclerosis. Lancet Neurol 19(6):481-482. https://doi. org/10.1016/S1474-4422(20)30147-2

66. Akhoundi FH, Sahraian MA, Moghadasi AN (2020) Neuropsychiatric and cognitive effects of the COVID-19 outbreak on multiple sclerosis patients. Mult Scler Relat Disord 41:102164. https://doi. org/10.1016/j.msard.2020.102164 
67. Racke MK, Newsome SC (2020) Multiple sclerosis and COVID19: a great opportunity for databases promoting research and collaboration. J Neuroimmunol 345:577283. https://doi. org/10.1016/j.jneuroim.2020.577283

68. Hartung HP, Aktas O (2020) COVID-19 and management of neuroimmunological disorders. Nat Rev Neurol. https://doi. org/10.1038/s41582-020-0368-9(Online ahead of print)

69. Shalhoub S (2020) Interferon beta-1b for COVID-19. Lancet 395(10238):1670-1671. https://doi.org/10.1016/S0140 $-6736(20) 31101-6$

70. Maghzi AH, Houtchens MK, Preziosa P, Ionete C, Beretich BD, Stankiewicz JM et al (2020) COVID-19 in teriflunomide-treated patients with multiple sclerosis. J Neurol. https://doi.org/10.1007/ s00415-020-09944-8

71. Bonavita S, Tedeschi G, Atreja A, Lavorgna L (2020) Digital triage for people with multiple sclerosis in the age of COVID-19 pandemic. Neurol Sci 41(5):1007-1009. https://doi.org/10.1007/ s10072-020-04391-9

72. Frischer JM, Bramow S, Dal-Bianco A, Lucchinetti CF, Rauschka $\mathrm{H}$, Schmidbauer M et al (2009) The relation between inflammation and neurodegeneration in multiple sclerosis brains. Brain 132:1175-1189

73. Berger JR, Brandstadter R, Amit Bar-Or A (2020) COVID-19 and MS disease-modifying therapies. Neurol Neuroimmunol Neuroinflamm 7:e761. https://doi.org/10.1212/NXI.000000000000076 1

74. David Bakera D, Amora S, Kanga A, Schmierera K, Giovannonia $\mathrm{G}$ (2020) The underpinning biology relating to multiple sclerosis disease modifying treatments during the COVID-19 pandemic. Multi Sclerosis Related Dis 43:102174 